\title{
Impact du mode de prélèvement sur la faune de l'Unité Forestière d'Aménagement (UFA) MOKABI-DZANGA au nord du Congo Brazzaville
}

\author{
Pierre MBETE ${ }^{1}$, Paul MOPOUNDZA ${ }^{1}$, Roger MBETE ${ }^{1}$, MASSANG-BIENET $^{1}$ et Christophe NGOKAKA $\dagger$ \\ Laboratoire des Productions Animales et de l'Environnement, École Nationale Supérieure d'Agronomie et de \\ Foresterie. Université Marien NGOUABI B.P. 69 BRAZZAVILLE \\ *Auteur correspondant : Pierre MBETE E-mail : mbete_pierre@yahoo.com, Tel : (242)066611898/053252825
}

Original submitted in on $17^{\text {th }}$ January 2014. Published online at www.m.elewa.org on $31^{\text {st }}$ March 2014. http://dx.doi.org/10.4314/jab.v75i1.6

\section{RESUME}

Objectif : L'objectif général de la présente étude consistait à évaluer les dégâts occasionnés par les opérations de l'exploitation forestière sur la faune sauvage dans l'Unité Forestière d'Aménagement (UFA) de MOKABI-DZANGA, notamment dans l'Assiette Annuelle de Coupe (AAC) 2010 au Nord du Congo. Les objectifs spécifiques dont le but est de gérer durablement les ressources naturelles dans une unité d'exploitation forestière se résument à déterminer

- i i les groupes d'espèces animales les plus prélevés par sexe, et par l'importance de la pression de la chasse sur les différentes espèces des Céphalophes ;

- $i_{2}$ les quantités et la répartition du stock de gibier prélevé selon l'état de conservation,

- $i_{3}$ l'importance relative du piégeage et du fusil.

Méthodologie et résultats : Une équipe de 15 chasseurs et 15 commerçants de viande de brousse étaient identifiés dans les villages riverains était sélectionnée pour atteindre nos objectifs. II ressort de nos résultats que, le groupe d'espèce de Céphalophus représente $(70 \%)$ était plus menacé suivi des petits primates $(10 \%)$, des Suidaes (5\%) et Rongeurs (3\%). Les enquêtes menées révèlent qu'une biomasse globale de $4359,50 \mathrm{~kg}$ était prélevée. La viande fumée avait un pourcentage très élevé $(66,95 \%)$ que la viande fraiche $(33,05 \%)$. Ainsi, les commerçants de cette activité ont une préférence sur la viande fumée que sur la viande fraiche. Par conséquent, la répartition par sexe indique que les mâles étaient plus abattus que les femelles. Les adultes faisaient l'objet d'un prélèvement plus important suivi des sub-adultes puis des vieux. Parmi les espèces, le Cephalophus callipygus était plus abattu suivi du Cephalophus monticola, du Cephalophus dorsalis, du Cephalophus sylvicultor et du Cephalophus nigrifons. De plus, on constate que la pratique des pièges est plus importante que celle du fusil.

Conclusion et application of résultats : Les résultats de cette étude montrent que l'ouverture des pistes forestières par l'exploitant occasionne une hémorragie désastreuse de la biomasse faunique. Des efforts doivent être entrepris par la société forestière propriétaire de I'UFA en vue d'améliorer et de minimiser l'impact des opérations forestières sur la faune.

Mots clés : Faune sauvage, Céphalophes, Chasse, Impact, Assiette Annuelle de Coupe, Mokabi-Dzanga. 


\section{Impact of sampling methods on the wildlife of the Forest Management Unit (FMU) MOKABI-DZANGA north of Congo Brazzaville}

Objective: The overall objective of this study was to assess the damage caused by the exploitations of the forest on wildlife in the Forest Management Unit (FMU ) of MOKABI - DZANGA, including the Annual Plate Cup ( AAC ) 2010 in northern Congo. The specific objectives which aim to sustainably manage natural resources in a unit of forest exploitation can be summarized as determining:

- $i_{1}$ the species groups most collected by sex, and the importance of hunting pressure on the different species of duiker ;

- $i_{2}$ quantity and distribution of the stock of game taken by the state of conservation,

- $i_{3}$ the relative importance of the traditional trap (to catch) and gun( to kill) animals.

Methodology and Results: A team of 15 hunters and 15 traders bushmeat were identified in the surrounding villages were selected to achieve these goals. It is clear from these results that the species Cephalophus group $(70 \%)$ was the most threatened, followed by small primates (10\%) Suidaes (5\%) and rodents (3\%). Investigations show that an overall biomass of $4359.50 \mathrm{~kg}$ was collected. Smoked meat had a higher preference $(66.95 \%)$ than fresh meat $(33.05 \%)$. By the sex , males were more killed than females. During hunts, it was that observed that the most animals killed in range, were adult and elder animals. Among the species, Cephalophus callipygus (Peter's duiker) was more shot followed Cephalophus monticola (Blue duiker), the Cephalophus dorsalis (Bay Duiker), f Cephalophus sylvicultor (Yellow-Backed Duiker) and Cephalophus nigrifons (Black -fronted Duiker) In these circumstances, it was confirmed that the trap practice was most of used than gun.

Conclusion and application of Results: The results of this study show that the opening of forest trails by the operator causes a disastrous impact on Wildlife biomass. Efforts should be undertaken by the forestry company owner UFA to improve and minimize the impact of forestry operations on wildlife.

Key-words: wild fauna, Cephalophes, hunt, damages, annual area cutting MOKABI-DZANGA

\section{INTRODUCTION}

Les besoins de plus en plus croissants du gouvernement congolais de créer des emplois à travers le territoire national ont permis l'ouverture de plusieurs zones à l'exploitation forestière au Nord Congo, alors que cette zone il y a 20 ans de cela était encore totalement enclavée laissant ainsi une chance au développement de la ressource naturelle et de la faune sauvage. Ainsi, plusieurs Unités Forestières d'Aménagement (UFA) ont été attribuées à des grandes sociétés industrielles telles que la Congolaise Industrielle des Bois (CIB), MOKABI SA, I'Industrie Forestière de OUESSO (IFO) qui exploitent, transforment et commercialisent le bois et les produits dérivés. Mais, si ces industries forestières sont d'importantes sources d'emplois et de revenus dans ces zones rurales, n'en demeure pas moins qu'elles apparaissent d'autre part comme de puissants moyens de destruction des ressources naturelles en générales et de la faune sauvage en particulier (Auzel, 1995). En effet, ces chantiers forestiers ouvrent des voies d'accès à l'intérieur des forêts et facilitent ainsi l'entrée des chasseurs dans les profondeurs des forêts donc, plus près des espèces animales (Auzel, 1999, Moukassa, 2004). D'autre part, ces chantiers forestiers attirent de plus en plus des populations tant rurales que urbaines ce qui à court et moyen terme peut poser des problèmes pour leur approvisionnement en ressources naturelles principalement les protéines d'origine animales (Moukassa, 2002). Aussi, l'on présume que, entre autres, l'accroissement de la population, la précarité des conditions de vie dans les bases vie seraient les inducteurs de la forte augmentation des prélèvements de gibiers dans les concessions forestières (Auzel, 1995). Ces prélèvements entraineraient sans doute le recule ou la disparition de la faune empêchant ainsi à 
cette faune d'assurer la régénération de la forêt notamment dans la dispersion des diaspores (Ngokaka et al, 2010). Ainsi, pour nous rendre compte une enquête a été menée auprès d'un échantillon représentatif de chasseurs et de commerçants de gibier de la zone couverte par l'étude. Les objectifs spécifiques consistaient à connaître :

- les groupes d'espèces animales par sexe les plus prélevés ainsi que l'importance relative de la pression de la chasse sur les différentes espèces des Céphalophes abattus au sein de

\section{METHODOLOGIE}

Matériel : Pour une bonne récolte des données sur le terrain, le matériel suivant a été utilisé :

- $\quad$ une carte de l'Assiette Annuelle de Coupe de I'UFA

- une carte de pistage de la zone d'étude,

- un GPS,

- une boussole

- $\quad$ une balance « Pesola » 50kg,

- $\quad$ une clé de détermination de type : Mammifères d'Afrique et de Madagascar (Haltenorth, 1977) et The kingdon Field to Africain Mammals (Kingdon, 1997),

- $\quad$ un tableau de Moss (1996).

Méthode de collecte des données : Une enquête a été menée auprès d'un échantillon représentatif de chasseurs et de commerçants de gibier de la zone couverte par l'étude. Au cours de l'étude, la carte de l'assiette annuelle de coupe et la carte de pistage de la zone ont servi au repérage des zones de chasse de I'UFA Mokabi-Dzanga Les observations étaient faites entre 7 heures et 17 heures pendant cinq 5 mois. Nos

\section{RESULTATS}

Estimation des groupes d'espèces animales les plus prélevés : La figure 1, montre les groupes d'espèces animales abattus dans la zone d'étude. II l'unité forestière d'aménagement de MokabiDzanga,

- $\quad$ les quantités du gibier prélevé,

- répartition du stock de gibier prélevé selon l'état de conservation,

- l'importance relative du piégeage et du fusil.

Les résultats de cette étude nous permettront de faire des propositions sur les possibilités de réduction la pression sur la faune sauvage dans l'unité forestière d'aménagement Mokabi-Dzanga.

enquêtes étaient menées sur un échantillon de quinze 15 personnes identifiées comme chasseurs et 15 comme commerçants intervenant dans la vente de gibiers au marché local. Les personnes enquêtées étaient questionnées afin d'obtenir des données qualitatives et quantitatives des gibiers prélevés. Ici, nous prenons toutes les données biologiques sur les gibiers, à savoir : le nom et le nombre d'espèce, l'âge et le sexe, le poids. Une mention spéciale était faite sur la technique de chasse (piège ou fusil calibre 12. Une clé de détermination de type : Mammifères d'Afrique et de Madagascar (Haltenorth, 1977) et The kingdon Field to Africain Mammals (Kingdon, 1997), était utilisée en vue d'identifier l'espèce animale. Pour estimer la structure des classes d'âge du gibier, on a fait recours au tableau de Moss (1996).

Traitement des données : Le traitement des données s'est effectué par le biais des logiciels suivants : ArcView 3.3, ArcGis 9.2, Excel, Map Source.

s'agit principalement des Céphalophes, suivis des petits primates, des suidés et des rongeurs. Les autres groupes sont faiblement prélevés. 


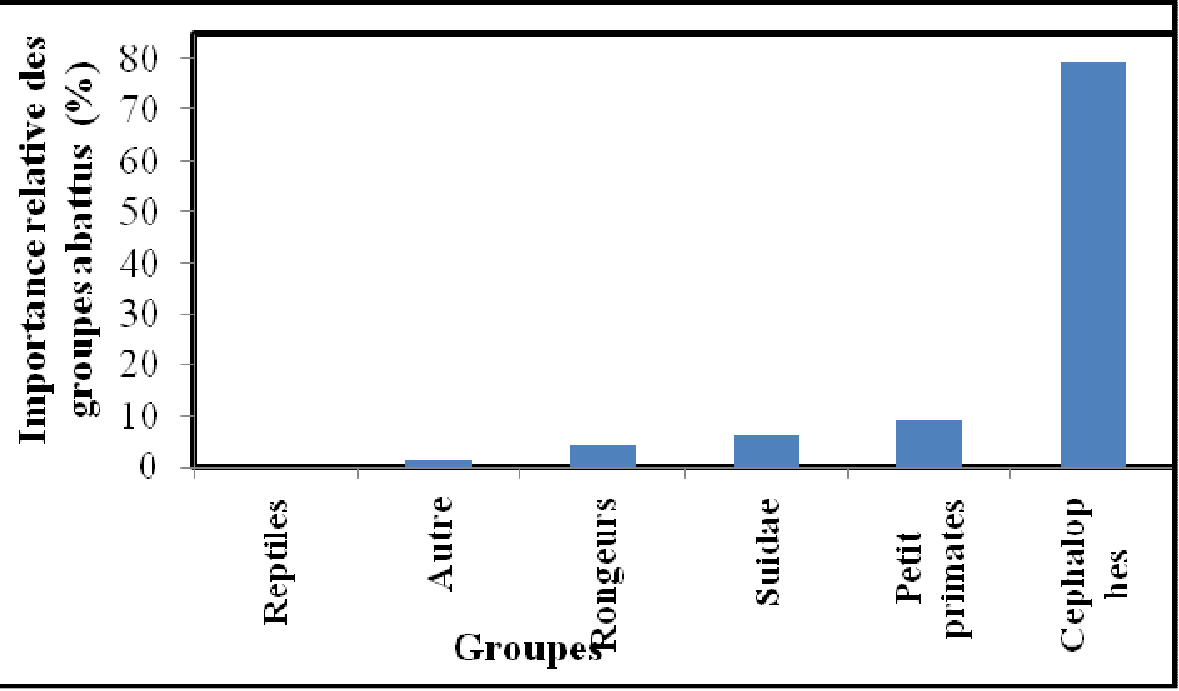

Figure 1 : Estimation relative des groupes d'espèces abattus

Estimation de la quantité du gibier prélevée : Las enquêtes menées dans la zone d'étude relèvent que sur 1051 prélèvements, on a obtenu une biomasse globale de $4359,50 \mathrm{~kg}$. Cette biomasse se répartit, selon l'importance, ainsi qu'il suit :

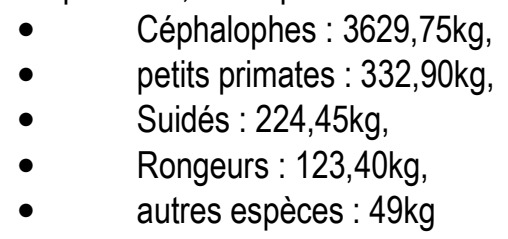

Répartition du stock de gibier selon l'état de conservation : Lors de la collecte des données dans les villages enquêtés, nous avons enregistré deux catégories de viande de chasse à savoir : la viande fraiche et la viande fumée, tableau1. L'examen du tableau 1 montre que :

- l'espèce Cephalophus monticola représente $31 \%$ de viande abattue, suivi de l'espèce
Cercopithecus nictitans 12\%, de Cephalophus dorsalis $8,4 \%$,

- $\quad$ Pour la viande fumée l'espèce Cephalophus dorsalis représente $8,1 \%$ suivi du Cephalophus sylvicultor 6,3\%, du Cercopithecus nictitans 4,3\% et du Cephalophus monticola 2,6\%.

Le gibier abattu est embarquée des villages sous deux états comme le confirme la figure 2 .

La figure 2 montre l'importance des différents états du gibier embarqué des villages enquêtés. Ainsi, nous avons enregistré un pourcentage plus élevé de gibier fumé $(66,95 \%)$ contre $(33,05 \%)$ pour le gibier frais.

Sur un prélèvement de 1051, on a identifié les sexes de 230 individus représentant $56,3 \%$ de mâles et $43,7 \%$ de femelles identifiés parmi les individus capturés à l'état frais, tableau 2.

Tableau 1 : Répartition du stock selon l'état de conservation.

\begin{tabular}{|l|l|l|l|l|l|}
\hline Nom pilote & Nom scientifique & Nom lingala & $\%$ frais & $\%$ fumé & Total \% \\
\hline Céphalophe à dos jaune & Cephalophus sylvicultor & Bemba & 1.9 & 6.3 & 8.2 \\
\hline Céphalophe à front noir & Cephalophus nigrifons & Djombe & 0.6 & 1.4 & 2.0 \\
\hline Civette & Viverra civetta & Edjoi & 0.3 & 0.0 & 0.3 \\
\hline Céphalophe baï & Cephalophus dorsalis & Gbomou & 8.4 & 8.1 & 16.5 \\
\hline Pangolin à longue queue & Manis tricuspis & kaka & 0.6 & 1.0 & 1.6 \\
\hline Cercopithèque à nez blanc & Cercopithecus nictitans & Koi & 12.4 & 4.3 & 16.7 \\
\hline Cercopithèque pogonias & Cecopithecus pogonias & Mambe & 2.2 & 0.0 & 2.2 \\
\hline Nandinie & Nandinia binotata & Mbala & 0.6 & 0.0 & 0.6 \\
\hline Chévrotain aquatéque & Hyemoschus aquaticus & Mbenguene & 1.0 & 0.8 & 1.8 \\
\hline Céphalophe bleu & Cephalophus monticola & Mboloko & 31.1 & 2.6 & 33.7 \\
\hline
\end{tabular}


Mbete et al. J. Appl. Biosci. 2014. Impact du mode de prélèvement sur la faune de l'UFA Mokabi-
Dzanga du Congo Brazzaville

\begin{tabular}{|c|c|c|c|c|c|}
\hline Sitatunga & Tragelaphus spekei & Mbouli & 1.2 & 0.2 & 1.4 \\
\hline Cercocèbe à joues grises & Locephocebus albigena & Ngada & 0.6 & 0.2 & 0.8 \\
\hline Mangouste à queue blanche & Herpestes icheneumon & Nganda & 3.1 & 0.7 & 3.8 \\
\hline Céphalophe de Peters & Cephalophus callipygus & Ngandi & 4.0 & 2.6 & $8 ; 6$. \\
\hline Crocodile des marais & Osteolaemus tetraspis & Ngoki & 0.3 & 0.2 & 0.5 \\
\hline Athérure & Atherurus africanus & Ngomba & 0.0 & 0.3 & 0.3 \\
\hline Potamochère & Potamochoerus porcus & Ngoulou & 0.25 & 0.25 & 0.5 \\
\hline Céphalophe à ventre blanc & Cephalophus leucogaster & Sengue & 0.0 & 0.5 & 0.5 \\
\hline Total & & & & & 100.00 \\
\hline
\end{tabular}

Tableau 2 : Répartition des gibiers abattus selon le sexe.

\begin{tabular}{|l|c|c|}
\hline Sexe & Quantité $\mathbf{( k g )}$ & Pourcentage (\%) \\
\hline Femelle & 98 & 43,7 \\
\hline Mâle & 132 & 56,3 \\
\hline Total & 230 & 100 \\
\hline
\end{tabular}

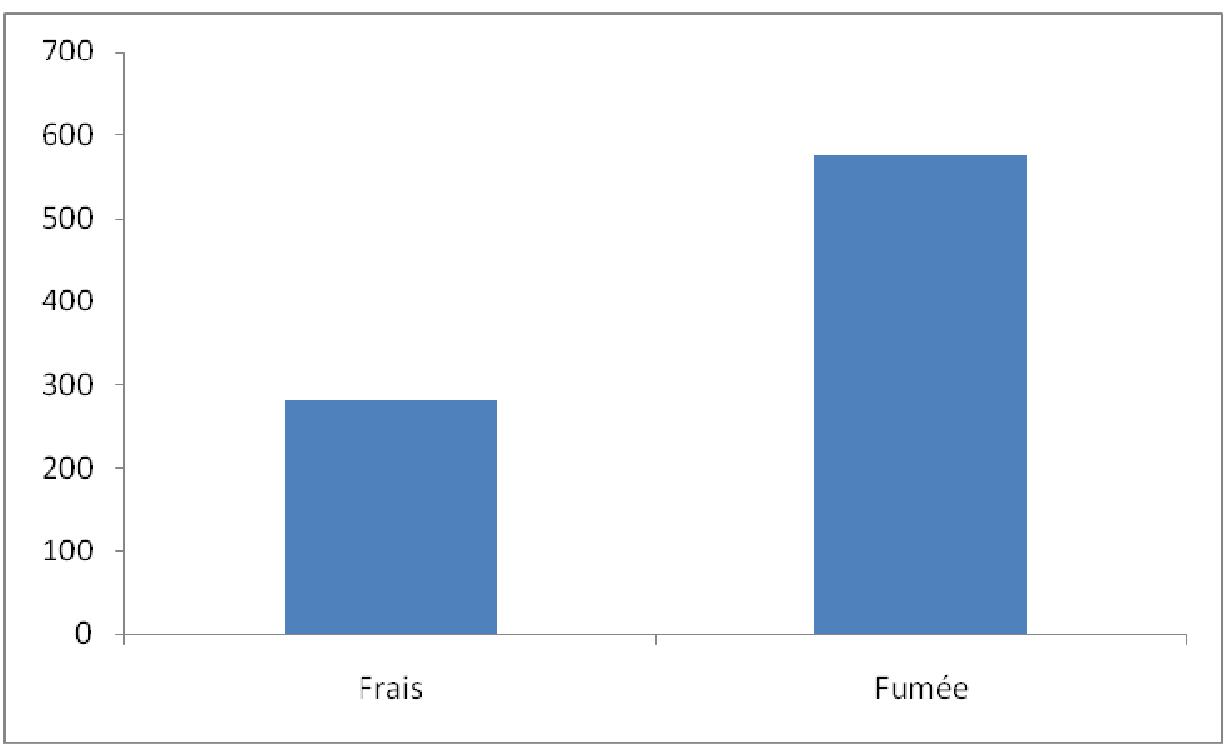

Figure 2 : Importance relative de l'état du gibier embarqué des villages enquêtés.

Importance relative de la pression de la chasse sur les Céphalophe : Les Céphalophes constituent en grande partie des espèces les plus chassées. La pression de la chasse par rapport à cette espèce a été évaluée à partir de la lecture de l'usure de la dentition. C'est une méthode qui consiste à comparer le model standard de Eaton (2002) avec l'usure de la dentition sur la mâchoire inférieure de l'espèce abattue. Après le dépouillement des données de la lecture de la dentition sur les mandibules que nous avons rangées on a identifié quatre classes (enfant, sub-adulte, adulte, vieux) figure 3. Cette figure montre l'importance des céphalophes abattus en fonction des classes d'âges. Elle montre que la classe de céphalophes la plus chassée est la classe des adultes viennent ensuite la classe sub-adulte et la classe des vieux. 


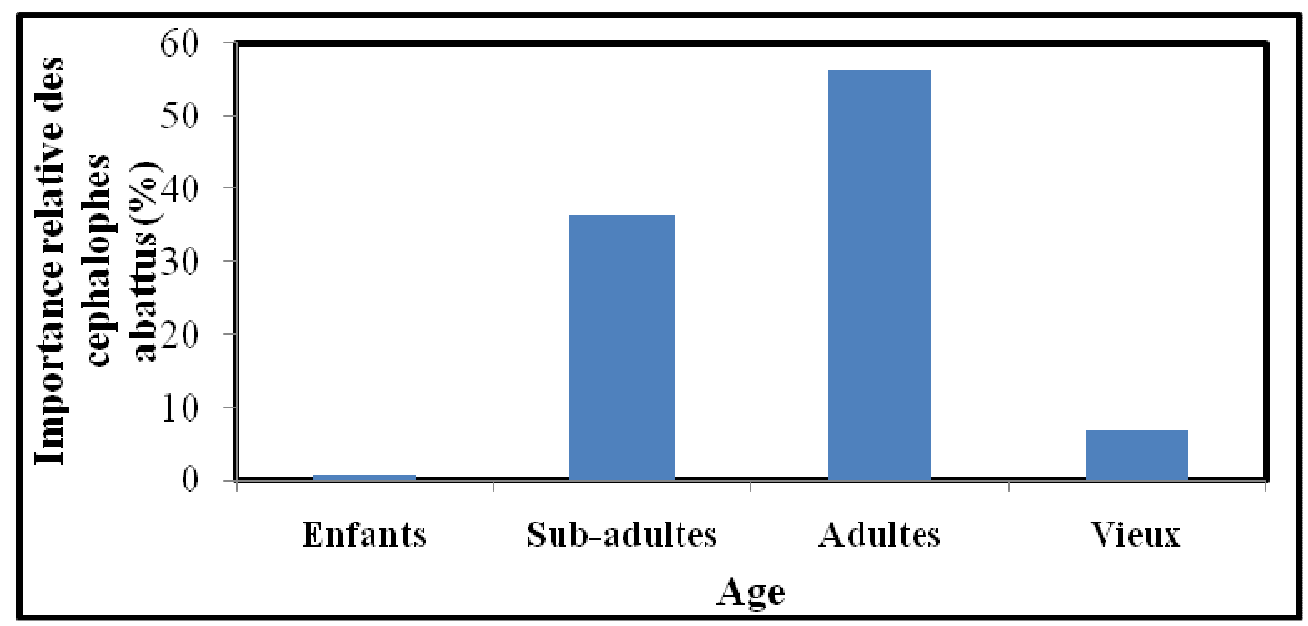

Figure 3 : Importance relative des classes d'âges des Céphalophes abattus

Répartition relative des différentes espèces de Céphalophes abattus : Le genre Céphalophe regroupe six espèces. Ainsi sur une biomasse totale de $4359,50 \mathrm{~kg}$ ce genre représente une biomasse de $3624,75 \mathrm{~kg}$. Cependant parmi les six espèces, le
Céphalophus callipygus est l'espèce la plus abattue, suivi du Céphalophus monticola, du Céphalophus dorsalis, du Céphalophus syvicultor, du Céphalophus nigrifons, figure 4.

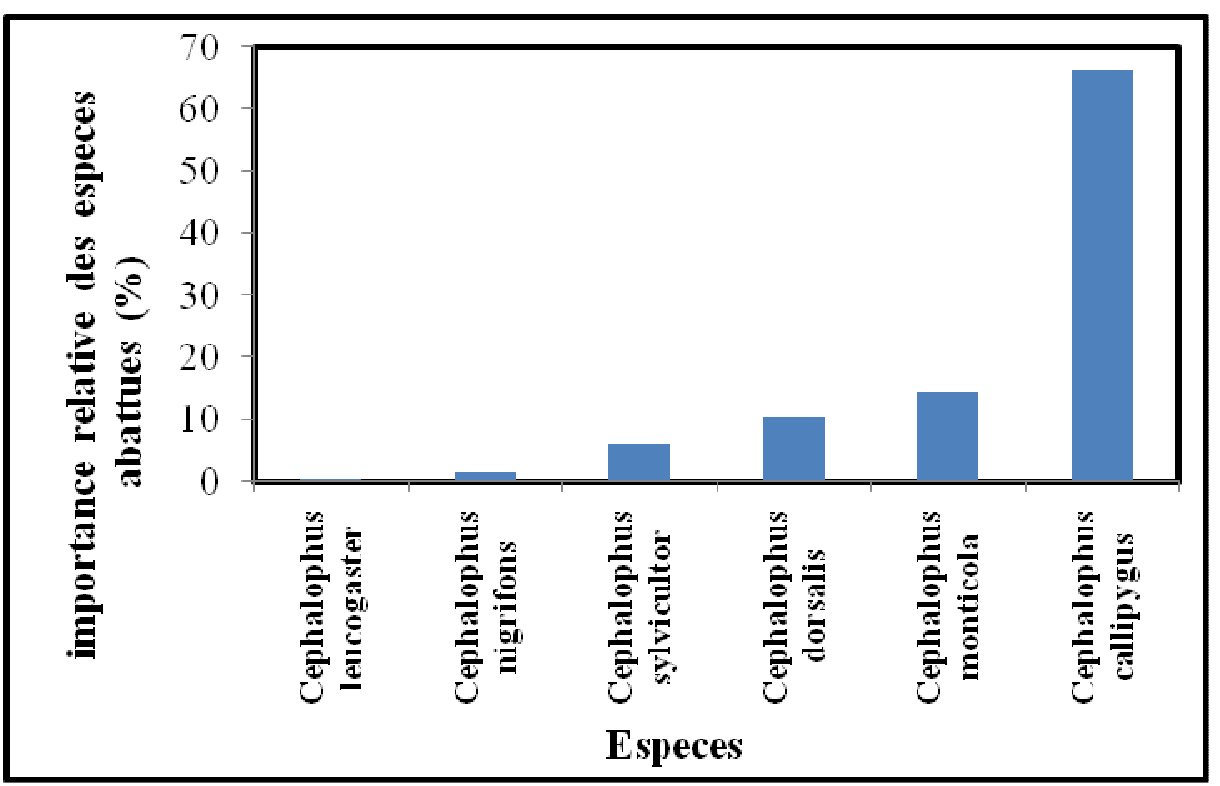

Figure 4 : Répartition relative des différentes espèces de céphalophes abattus

Importance relative du piégeage et du fusil : Les techniques de chasse les plus utilisées dans la zone d'étude sont les pièges aux câbles métalliques et le fusil. La chasse au piège est pratiquée sous deux formes. La première forme la plus utilisée : c'est le piégeage où l'animal est capturé par les pattes. Cette forme se pratique toute l'année de façon permanente. Elle vise surtout les gros gibiers. Un chasseur peut compter environ deux cent pièges. La deuxième forme appelée "Garden huntig 》 vise particulièrement I'Atherure et se pratique surtout la saison pluvieuse. Ici le piège capture l'animal à travers son cou ou le reste de l'abdomen. La chasse au fusil est la technique la plus efficace pour les grands braconniers. Elle est pratiquée surtout la nuit et rarement au couchée du soleil. Elle existe sous deux formes ou modes ; soit la 
chasse est organisée par un commanditaire qui est souvent un commerçant, un trafiquant, un fonctionnaire et bien d'autres qui fournissent aux chasseurs les moyens nécessaires à savoir : l'arme, les cartouches, les vivres, la torche, les piles. La deuxième forme, soit les chasseurs s'organisent entre eux en formant une équipe de cinq ou plus, utilisent leurs moyens afin de mener à bien leur partie de chasse. Ici, les chasseurs aménagent des campements de chasse près des pistes forestières et patrouillent sans relâche les placettes de comptage des arbres afin de maximiser les chances de captures. Les quantités de gibiers prélevés selon les deux techniques de chasse sont représentées dans la figure 5. L'examen de la figure 5 montre que $72 \%$ du gibier étaient prélevés au moyen des câbles contre $28 \%$ tués par le fusil.

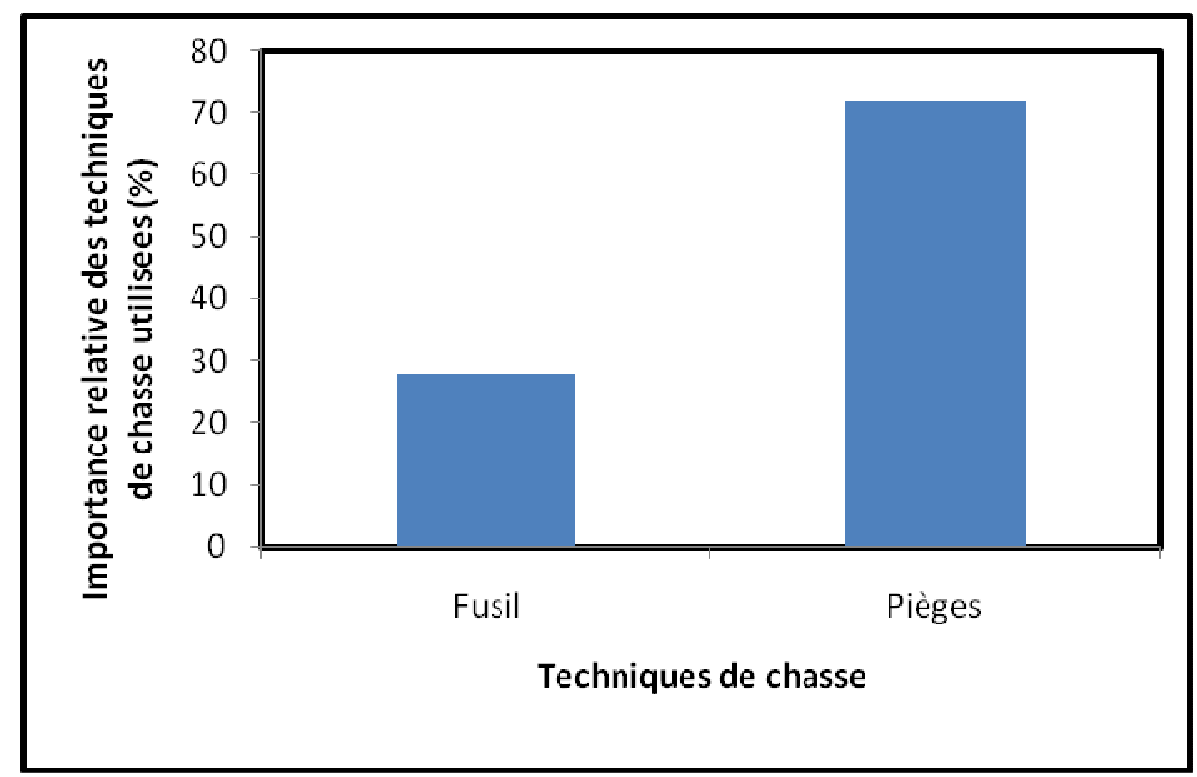

Figure 5 : Importance relative du piégeage et du fusil

\section{DISCUSSION}

Le traitement des résultats montre que les Céphalophes sont les espèces les plus chassées. Plusieurs raisons peuvent être à l'origine de cette situation. En premier lieu, on peut penser à l'abondance dans notre zone d'étude des individus appartenant à ce groupe. En effet, il est évidentes que grâce à leur abondance, certaines espèces sont plus facilement visibles que d'autres et en conséquence, peuvent être abattues par les chasseurs (Auzel, 1995). Au-delà de l'abondance, la qualité du gibier peut aussi justifier la forte pression des chasseurs sur ce groupe (Ngokaka et al, 2010). En effet, le commerce de la viande de chasse fait intervenir plusieurs acteurs parmi lesquels : les commanditaires, les chasseurs, les vendeurs et les consommateurs. Pour des raisons de rentabilité de leurs affaires, les commanditaires scrutent les différents marchés et en fonction des informations reçues sur la qualité du gibier qui est plus appréciée par les vendeurs et les consommateurs, ils passent leurs commandes auprès des chasseurs qui ont l'obligation de ne chasser que ce qui figure sur leurs carnets de commande
(Ngokaka et al, 2010). Les espèces comme le Céphalophe bleu, Céphalophe de Peters, Céphalophe bai, Céphalophe à franc noir, très appréciées par les ménages congolais en général et ceux du Département de la Likouala en particulier, paient ainsi une tribu pour la qualité de leur viande (Ngokaka et al, 2010). En ce qui concerne la quantité de gibier prélevée, on note que les Céphalophes sont encore le groupe qui fournit la plus grande biomasse. Ce résultat confirme la pression de la chasse sur ce groupe dans notre zone d'étude. Pour ce qui est de l'état de stock de conservation, il est évident que les paysans accourent beaucoup plus à l'état fumé. En effet, indépendamment du fait que le gibier fumé se conserve plus longtemps, dans ces zones rurales, les paysans ne disposent d'aucun moyen leur permettant de conserver du gibier frais ce qui leur permettrait de vendre de la viande fraiche plus chère surtout en période de pénurie. S'agissant de la répartition des individus prélevés par sexe, on constate que les individus mâles sont plus abattus que les femelles. Deux raisons fondamentales peuvent justifier 
ces prélèvements : En premier lieu, il est bien connu que le mâle, peu importe l'état physiologique dans lequel il se trouve, reproduction ou repos sexuel, est toujours plus actif que la femelle (Ngokaka et al, 2010). Cela s'observe surtout chez les animaux nomades ou qui vivent en couple. Ainsi le mâle, chef de famille, explore souvent l'environnement pour satisfaire les besoins du troupeau ou du couple. A cette occasion, devenant plus visible, il peut facilement être pris dans un piège ou être abattu par le chasseur (Ngokaka et al, 2010). Ensuite, les chasseurs à l'affût, recherchent souvent les plus beaux spécimens, les phénotypes rares. Or souvent, ce sont les mâles qui sont porteurs de ces caractères. D'où leur fort taux de prélèvement

\section{CONCLUSION}

A travers cette étude, nous venons de montrer que l'installation d'une industrie forestière est à l'origine d'une exploitation massive de la faune sauvage. Les conséquences immédiates sont que, certaines espèces animales vont progressivement disparaître si aucune action n'est prise pour modifier l'offre et la demande en viande de chasse. Lorsqu'on connaît le rôle de la faune dans la régénération naturelle, ces disparitions vont entrainer les modifications de ces écosystèmes. On court donc le risque d'un développement à rebours de ces écosystèmes. Ici encore, il ne faut pas oublier que la forte pression exercée sur les mâles peut également

\section{REMERCIEMENTS}

Les auteurs remercient d'une part les responsables de la société MOKABI-DZANGA pour l'aide technique et matérielle qu'ils leur ont apportée et d'autre part le Réseau des Institutions de Formation Forestières et

\section{REFERENCES BIBLIOGRAPHIQUES}

Akouango P., Ngokaka C., Mbete P., and Vouidibio J., (2013). Assement of the quantities of sold games in the market of a lumbering yard in the north Congo. Case of the market of Ngombé. IJABPT. Volume -4. ISSN 0976-4550. pp 220225.

Auzel P., (1995). Evaluation de l'impact de la chasse sur la faune des forêts d'Afrique Centrale. Université Paris XII Val de Marne : 64p.

Auzel P., (1999). Sites forestiers industriels et durabilité de l'exploitation de la faune (dans le Sud-est du Cameroun. 120p.
(Ngokaka et al, 2010). En ce qui concerne l'importance relative à la pression des Céphalophes autour des concessions forestières, du piégeage et du fusil, nos résultats concordent avec ceux réalisés par Pierre Mbete et al, (2010) qui montrent que, les Céphalophes constituaient le groupe le plus prisé aux alentours du Parc National d'Odzala Kokoua. Le piégeage du gibier par rapport à la pratique du fusil reste le moyen le plus utilisé par les populations riveraines dans les zones forestières et ce résultat, corrobore avec les travaux de Pierre Mbete et al, (2010) autour du parc national d'odzala kokoua, ainsi à ceux de Parisse Akouango et al, (2013) autour du chantier forestier de Ngombé en République

du

Congo.

compromettre l'avenir de nombreuses espèces dans notre zone d'étude. En effet, en prélevant massivement les mâles, on risque de se retrouver uniquement avec les femelles qui seront à elles seules incapables de transmettre le patrimoine génétique de ces espèces aux générations futures. II est donc souhaitable que la réglementation en matière de chasse notamment en ce qui concerne les prélèvements des espèces tant sur le plan qualitatif que quantitatif soit respectée dans toute sa plénitude dans la zone de l'Unité Forestière d'Aménagement Mokabi-Dzanga.

Environnementale d'Afrique Centrale (RIFFEAC) pour l'aide financière qui a permis de réaliser la présente étude.

Auzel, P., (2001). Les villes en forêts: impact de l'exploitation forestière sur la gestion coutumière des ressources naturelles, in W.De1vingt, editor. La forêt des hommes terroir et forêts tropicales africaines. Presses Agronomiques de Gembloux, Gembloux, Belgique. p 235-251

Ekoungoulou T., 2010. Durabilité de la chasse villageoise : estimation des tendances et approche de gestion (UFA Ngombé), Université Marien Ngouabi, Mémoire de fin d'études.127p. 
Elende G. A. et Zoubabela A., (2008), Rapport préliminaire sur le suivi de la viande de brousse dans deux villages pilotes (Liouesso et Mokouangonda), WCS-PROGEP-PNOK. $78 \mathrm{P}$

Haltenorth, T., Diller, H. et Cuisin, M., (1977). Mammifères d'Afrique et de Madagacar. Delachaux et Niestlé, Editeurs, NeuchatelParis. 397p

Kingdon, (1997). The kingdon Field guide to Africain Mammals Academic Press, Oxford ISBN 0691-1 1 692-X 476p.

Mbete P., C. Ngokaka., F. Akouango., N. Bonazaba et J. Vouidibio., (2010). Evaluation des quantités de gibiers prélevées autour du Parc National d'Odzala-Kokoua et leurs impacts sur la dégradation de la biodiversité. Journal of Animal \& Plant Sciences, Vol 8. pp1061-1069

Moukassa, A., (2004). Se nourrir dans un camp forestier: Suivi de l'alimentation des ménages dans les sites forestiers de Kabo et de Ndoki 2, $p 12$,

Ngokaka C., Boukoulou H., Akouango F., Mbete P., Kandza V-H., \& J. Vouidibio (2010). Fréquentation de saline d'Ebobobo par les Gorilles : un atout pour la promotion $\mathrm{du}$ tourisme de vision en République du Congo. Journal of Animal \& Plant Sciences, Vol 9. pp1086-1093

Peters, (1993). Exploitation de la faune et développement rural (FAO-PNUE, $n^{\circ} 1$. vol 9) p13-15 